\title{
Effect of PPAR inhibition during pregnancy on posterior cerebral artery function and structure
}

\author{
Siu-Lung Chan ${ }^{1}$, Abbie C. Chapman ${ }^{1}$, Julie G. Sweet ${ }^{1}$, Natalia I. Gokina ${ }^{2,3}$ and Marilyn J. Cipolla ${ }^{1,2,3 *}$ \\ Department of Neurology, University of Vermont, Burlington, VT, USA \\ 2 Department of Obstetrics, Gynecology and Reproductive Sciences, University of Vermont, Burlington, VT, USA \\ ${ }^{3}$ Department of Pharmacology, University of Vermont, Burlington, VT, USA
}

\section{Edited by:}

R. C. Webb,

Medical College of Georgia, USA

\section{Reviewed by:}

R. C. Webb,

Medical College of Georgia, USA

Theodora Szasz,

Medical College of Georgia, USA

*Correspondence:

Marilyn J. Cipolla,

Department of Neurology, University of

Vermont, 89 Beaumont Ave., C454

Given, Burlington, VT 05401, USA.

e-mail:marilyn.cipolla@uvm.edu
Peroxisome proliferator-activated receptor- $\gamma$ (PPAR $\gamma$ ), a ligand-activated transcription factor, has protective roles in the cerebral circulation and is highly activated during pregnancy. Thus, we hypothesized that PPAR $\gamma$ is involved in the adaptation of cerebral vasculature to pregnancy. Nonpregnant (NP) and late-pregnant (LP) rats were treated with a specific PPAR $\gamma$ inhibitor GW9662 (10 mg/kg/day, in food) or vehicle for 10 days and vascular function and structural remodeling were determined in isolated and pressurized posterior cerebral arteries (PCA). Expression of PPAR $\gamma$ and angiotensin type 1 receptor (AT1R) in cerebral (pial) vessels was determined by real-time RT-PCR. PPAR $\gamma$ inhibition decreased blood pressure and increased blood glucose in NP rats, but not in LP rats. PPAR $\gamma$ inhibition reduced dilation to acetylcholine and sodium nitroprusside in PCA from NP ( $p<0.05$ vs. LP-GW), but not LP rats. PPAR $\gamma$ inhibition tended to increase basal tone and myogenic activity in PCA from NP rats, but not LP rats. Structurally, PPAR inhibition increased wall thickness in PCA from both NP and LP rats $(p<0.05)$, but increased distensibility only in PCA from NP rats. Pregnancy decreased expression of PPAR $\gamma$ and AT1R $(p<0.05)$ in cerebral arteries that was not affected by GW9662 treatment. These results suggest that PPAR $\gamma$ inhibition had significant effects on the function and structure of PCA in the NP state, but appeared to have less influence during pregnancy. Down-regulation of PPAR $\gamma$ and AT1R in cerebral arteries may be responsible for the lack of effect of PPAR $\gamma$ in cerebral vasculature and may be part of the vascular adaptation to pregnancy.

Keywords: peroxisome proliferator-activated receptor- $\gamma$, pregnancy, posterior cerebral arteries, vasodilation, vascular remodeling

\section{INTRODUCTION}

There are numerous hemodynamic changes that occur during pregnancy. For example, blood pressure decreases mid-pregnancy despite considerable expansion of blood volume and increased cardiac output (Gordon, 2007). The relatively low blood pressure in the face of increased plasma volume is likely due to reduced peripheral vascular resistance (Clapp and Capeless, 1997) and adaptation of the systemic vasculature to pregnancy that includes decreased responsiveness to vasoconstrictors (Parent et al., 1990; Pascoal et al., 1995), decreased sympathetic vasoconstriction (Ralevic and Burnstock, 1996), diminished myogenic tone (Learmont et al., 1996), and changes in vessel wall composition (Mackey et al., 1992). In addition, decreased vascular resistance increases blood flow in several organs during pregnancy, including renal and uterine circulations (Palmer et al., 1992; Carlin and Alfirevic, 2008). However, despite substantial hemodynamic changes in systemic and other organs during pregnancy, vascular resistance and blood flow in the brain appears to be unchanged (Williams and Wilson, 1998; Cipolla and Bullinger, 2008). Unlike systemic, renal, cardiac and uterine circulations where substantial hemodynamic changes are essential to accommodate increased blood volume and flow during pregnancy, the lack of hemodynamic changes in the brain is likely important in an organ that relies mainly on oxidative metabolism and needs relatively constant blood flow. Thus, it is possible that the adaptation of the cerebral circulation to pregnancy is to maintain normal vascular resistance and flow in the face of substantial changes in hemodynamics in other organ systems.

Despite apparently unchanged vascular resistance and blood flow in the brain (Williams and Wilson, 1998; Cipolla and Bullinger, 2008), there are considerable changes in the cerebral vasculature during pregnancy. For example, endothelium-derived hyperpolarizing factor-mediated dilation is lost whereas nitric oxide (NO) is prominent in cerebral arteries during pregnancy (Cipolla et al., 2004). In addition, cerebral arteries appear to adapt to the large amount of hormones produced during pregnancy as the constricting influence of pregnant plasma is normalized in cerebral arteries from pregnant rats (Amburgey et al., 2009). Further, cerebral (pial) arteries force dilatate at lower pressures during pregnancy (Cipolla et al., 2004). Pregnancy also appears to have a significant effect on hypertensive remodeling in cerebral vasculature. Pregnancy reverses and inhibits inward remodeling associated with hypertension, an effect that may maintain normal vascular resistance and blood flow during elevated blood pressure (Cipolla et al., 2006, 2008). Together, these adaptations may have an important protective role in the brain and contribute to the unchanged vascular resistance and blood flow during normal pregnancy. 
Although it appears the cerebral circulation adapts to pregnancy, the underlying mechanism by which this occurs is not clear. Peroxisome proliferator-activated receptor- $\gamma(\operatorname{PPAR} \gamma)$, a nuclear receptor transcription factor, regulates the transcription of target genes involved in adipogenesis, glucose homeostasis, and lipid metabolism (Berger et al., 2005). The role of PPAR $\gamma$ has also been implicated in pregnancy. PPAR $\gamma$ is highly activated during pregnancy (Waite et al., 2005) and has an important role in maternal metabolism, placental development, and trophoblast invasion (Wieser et al., 2008). Recently, the pleiotropic effect of PPAR $\gamma$ has been shown in the cardiovascular system. PPAR $\gamma$ appears to have direct protective effects including anti-hypertensive, antiinflammatory, and anti-atherosclerotic properties in the vasculature (Hsueh and Law, 2001; Robinson and Grieve, 2009; Sigmund, 2010). These protective effects of PPAR $\gamma$ may be more prominent in the cerebral circulation. For example, genetic interference of PPAR $\gamma$ function reduced the vasodilatory response to ACh in basilar arteries, but not in aorta (Beyer et al., 2008). The same study also showed that interference of PPAR $\gamma$ function causes hypertrophic inward remodeling, suggesting that inhibiting structural remodeling may also be a protective effect of PPAR $\gamma$ in cerebral vasculature. Thus, the role of PPAR $\gamma$ in the cerebral circulation appears similar to that of pregnancy, including enhanced $\mathrm{NO}$-dependent dilation and inhibition of structural remodeling. Considering PPAR $\gamma$ is highly activated during pregnancy, we hypothesized that PPAR $\gamma$ activity is involved in the vascular adaptation to pregnancy in the cerebral circulation. To test this hypothesis, pregnant and non-pregnant (NP) rats were treated with GW9662, a specific PPAR $\gamma$ inhibitor that acts through cysteine modification in the ligand-binding site of PPAR $\gamma$ (Leesnitzer et al., 2002). We investigated the effect of PPAR $\gamma$ inhibition during pregnancy on NO-mediated dilation, myogenic activity, and structural characteristics of posterior cerebral arteries (PCA). In addition, gene expression of PPAR $\gamma$ and angiotensin type 1 receptor (AT1R) were determined in cerebral arteries because of the potential involvement of these receptors in vascular function and remodeling during pregnancy (Cox et al., 1996).

\section{MATERIALS AND METHODS \\ ANIMALS AND TREATMENT GROUPS}

Pregnant (6-8 days pregnant) and NP (12-14 weeks old) Sprague Dawley rats (Charles River, Canada) were housed in the University of Vermont Animal Care Facility. Animals were randomly selected and treated with either GW9662 $(10 \mathrm{mg} / \mathrm{kg} /$ day for 10 days in food; $n=8)$ or vehicle $(n=8)$. This dose of GW9662 was higher than has been reported in other studies (Sivarajah et al., 2005) due to the higher activation of PPAR $\gamma$ in pregnancy (Waite et al., 2000). Rats were studied on day 20 or 21 of pregnancy. There was one NP rat that did not consume GW9662, and thus was excluded from the study and all comparisons. All procedures were approved by the Institutional Animal Care and Use Committee and conducted in accordance with the National Institute of Health Guide for the Care and Use of Laboratory Animals.

\section{BLOOD PRESSURE AND GLUCOSE MEASUREMENTS}

Blood pressures were measured non-invasively every 2-3 days during GW9662 treatment by determining the tail blood volume with a volume pressure recording sensor and an occlusion tail cuff
(Coda 8 System, Kent Scientific, Torrington, CT, USA), as previously described (Cipolla et al., 2008). Briefly, the animals were placed in individual holders and both the occlusion cuff and the volume pressure recording cuff were secured close to the base of the tail. The Coda 8 system measures systolic, diastolic, and mean blood pressures. Blood samples were collected from the tail of conscious rats for blood glucose measurement on the last day of the treatment, prior to euthanization (FreeStyle Lite, Abbott Inc., Alameda, CA, USA).

\section{VESSEL PREPARATION AND PRESSURIZED ARTERIOGRAPH SYSTEM}

Animals were anesthetized with isoflurane (3\% in oxygen), decapitated, and the brain was quickly removed and placed in cold HEPES-buffered physiological salt solution. A third-order branch of the PCA was carefully isolated and mounted on glass cannulas in an arteriograph chamber, the proximal cannula of which was connected to an in-line pressure transducer and a servo-null pressure control system (Living Systems Instrumentation, Burlington, VT, USA). The distal cannula was closed during the experiment to avoid flow-mediated responses. Temperature and $\mathrm{pH}$ were continually measured and maintained at $37.0 \pm 0.5^{\circ} \mathrm{C}$ and $7.40 \pm 0.05$, respectively. Measurements of inner diameter (ID) and wall thickness (WT) were made via video microscopy (Living Systems Instrumentation, Burlington, VT, USA). The remaining cerebral pial vessels were dissected from the brain, collected in RNAlater (Qiagen Inc, Valencia, CA, USA) and rapidly frozen for PCR analysis.

\section{EXPERIMENTAL PROTOCOL}

Arteries were equilibrated for $1 \mathrm{~h}$ at $25 \mathrm{mmHg}$. Active myogenic activity was determined by stepwise increases in pressure (in $25 \mathrm{mmHg}$ increments) from 25 to $175 \mathrm{mmHg}$ and ID measured at each pressure. To obtain agonist-induced vasodilatory responses, pressure was returned to $75 \mathrm{mmHg}$ until tone developed and diameter stabilized. Arteries were then exposed to cumulative concentrations of ACh $\left(10^{-8}-10^{-5} \mathrm{M}\right)$ and ID recorded at each concentration once stable. ACh was then washed from the bath and SNP $\left(10^{-8}-10^{-5} \mathrm{M}\right)$ was cumulatively added and ID recorded. To obtain structural measurements, ID and WT were recorded at pressures between 5 to $175 \mathrm{mmHg}$ after papaverine $\left(10^{-4} \mathrm{M}\right)$ and diltiazem $\left(10^{-5} \mathrm{M}\right)$ were added to the bath to fully relax smooth muscle.

\section{REAL-TIME RT-PCR ANALYSIS OF TARGET GENES IN PIAL VESSELS}

Gene expression of PPAR $\gamma$ and AT1R were determined in cerebral vessels using standard techniques for real-time RT-PCR, as described previously (Cipolla et al., 2009). These procedures were performed by the DNA facility at the University of Vermont. Results were calculated by the $2^{-\Delta \Delta \mathrm{CT}}$ method (Livak and Schmittgen, $2001)$. The percent change in gene expression was normalized to an endogenous gene $\left(\beta_{2}\right.$-microglobulin, B2M) and relative to the untreated NP group.

\section{DRUGS AND SOLUTIONS}

ACh, SNP, papaverine, and diltiazem were purchased from Sigma (St. Louis, MO, USA). Drugs were made as stock solutions each week and stored at $4^{\circ} \mathrm{C}$. GW9662 was purchased from Cayman Chemical (Ann Arbor, MI, USA). All vessel experiments were 
performed in physiological salt solutions containing the following (in $\mathrm{mM}$ ): $142.0 \mathrm{NaCl}, 4.7 \mathrm{KCl}, 1.2 \mathrm{MgSO}_{4}, 0.5 \mathrm{EDTA}, 2.8 \mathrm{CaCl}_{2}$, 10.0 HEPES, $1.2 \mathrm{KH}_{2} \mathrm{PO}_{4}$, and 5.0 glucose.

\section{DATA CALCULATIONS AND STATISTICAL ANALYSIS}

Percent tone was calculated as percent decrease in ID from the passive diameter at each intravascular pressure: [1 - $\left(\mathrm{ID}_{\text {active }}\right.$ ' $\left.\left.\mathrm{ID}_{\text {passive }}\right)\right] \times 100 \%$. Outer diameter $(\mathrm{OD})$ was calculated from measured ID and WT: ID + 2WT. Wall-to-lumen ratio was calculated as: ID/WT. Cross-sectional area (CSA) of the wall was calculated as: $\left[(0.5 \times \mathrm{OD})^{2}-(0.5 \times \mathrm{ID})^{2}\right] \times \pi$. Passive distensibility was calculated as the percent change in ID at a given pressure (ID ${ }_{p}$ ) from the original diameter $\left(\mathrm{ID}_{\mathrm{o}}\right.$, at $\left.5 \mathrm{mmHg}\right)$ : [( $\left.\left.\mathrm{ID}_{\mathrm{p}}-\mathrm{ID}_{\mathrm{o}}\right) / \mathrm{ID}_{\mathrm{o}}^{\mathrm{p}}\right] \times 100 \%$.

All data are presented as mean \pm SEM. Differences between individual groups were compared with one-way analysis of variance (ANOVA) with post hoc Student-Newman-Keuls Multiple Comparison Test, using Graph Pad Prism 5 (Graph Pad Software Inc., La Jolla, CA, USA). It was anticipated that pregnancy would alter responses to GW9662, thus two-way ANOVA was used to compare two independent variables GW9662 treatment and pregnancy, and their interaction. Differences were considered significant when $p<0.05$.

\section{RESULTS}

\section{PHYSIOLOGICAL PARAMETERS}

Physiological parameters for all groups of animals are shown in Table 1. Pregnancy significantly decreased blood glucose compared to the untreated NP group. GW9662 increased blood glucose in NP rats but had no effect on late-pregnant (LP) rats. Pregnancy decreased mean arterial pressure compared to untreated NP rats. GW9662 treatment decreased mean arterial pressure in NP rats, but did not further affect blood pressure in LP rats. As expected, body weights were significantly increased in LP rats. GW9662 did not affect body weights of either NP or LP rats.

\section{EFFECTS OF GW9662 DURING PREGNANCY ON TONE AND MYOGENIC ACTIVITY}

Figure 1 shows percent tone in PCA at $75 \mathrm{mmHg}$ from all groups. GW9662 treatment tended to increase tone in PCA from NP rats ( $44 \pm 3 \%$ vs. $30 \pm 6 \%$ in control, $p=0.08$ ), but had no effect in PCA from LP rats. Figure 2 shows active internal diameters versus intraluminal pressure for all groups of animals. Most PCA constricted when pressure increased from 25 to $75 \mathrm{mmHg}$, demonstrating

Table 1 | Physiological parameters of non-pregnant (NP), NP treated with GW9662 (NP + GW), late-pregnant (LP), and LP treated with GW9662 (LP+ GW).

\begin{tabular}{lccrc}
\hline & NP $(\boldsymbol{n}=\mathbf{8})$ & $\begin{array}{l}\mathbf{N P}+\mathbf{G W} \\
(\boldsymbol{n}=\mathbf{7})\end{array}$ & $\mathbf{L P}(\boldsymbol{n}=\mathbf{8})$ & $\begin{array}{l}\text { LP+GW } \\
(\boldsymbol{n}=\mathbf{8})\end{array}$ \\
\hline Body weights $(\mathrm{g})$ & $308 \pm 4$ & $306 \pm 5$ & $384 \pm 7^{*}$ & $381 \pm 5^{*}$ \\
Blood glucose $(\mathrm{mg} / \mathrm{dl})$ & $109 \pm 3$ & $132 \pm 9^{*}$ & $88 \pm 5^{*}$ & $91 \pm 4 \dagger$ \\
Mean arterial & $105 \pm 3$ & $92 \pm 4^{*}$ & $92 \pm 4^{*}$ & $87 \pm 3^{*}$ \\
pressure (mmHg) & & & & \\
\hline
\end{tabular}

Data are presented as means \pm SEM.

${ }^{*} p<0.05$ vs. NP; $+p<0.05$ vs. NP+GW. active myogenic activity that was sustained until $175 \mathrm{mmHg}$ (Figures 2A,B). At $175 \mathrm{mmHg}$, there was a dramatic increase in ID as arteries underwent forced dilatation. PCA from GW9662-treated NP rats had smaller ID at all pressures when compared to untreated NP rats, although this was not statistically significant (Figure 2A). The decreased ID was particularly obvious at 75 and $100 \mathrm{mmHg}$ (decreased by $23 \%$ and $24 \%$, respectively) when compared to untreated NP rats. This smaller ID may be due to increased tone by GW9662 treatment. In contrast to NP rats, GW9662 treatment did not decrease ID, but rather tended to increase ID of PCA at all pressures from LP rats, although this was not statistically significant.

\section{EFFECTS OF GW9662 DURING PREGNANCY ON ACh- AND SNP-INDUCED DILATION}

In the GW9662-treated NP group, four experiments were excluded because there were three PCA that either did not develop basal tone or maximally dilated prematurely during ACh exposure. In addition, there was one PCA in each untreated NP and LP group that did not develop tone, and thus were excluded. These explain the inconsistent and low number in GW9662-treated NP group $(n=4)$.

Figure 3A shows the concentration-response relationship of ACh-induced dilation. GW9662 inhibited ACh-induced dilation in PCA from NP rats but did not affect the response to ACh in PCA from LP rats. Two-way ANOVA revealed that dilator responses to ACh (from $3 \times 10^{-7}$ to $10^{-5} \mathrm{M}$ ) in PCA from NP-GW rats were significantly different from that of LP-GW rats. This difference was likely due to absence of dilation to ACh in PCA from NP-GW rats because GW9662 did not impact ACh-induced dilation in PCA from LP rats.

Figure 3B shows the concentration-response of SNP-induced dilation for all groups of animals. GW9662 treatment decreased the dilator response to SNP in PCA from NP rats, although this was not statistically significant, likely due to the small sample size in NP-GW group. Similar to the dilator response to ACh, two-way ANOVA

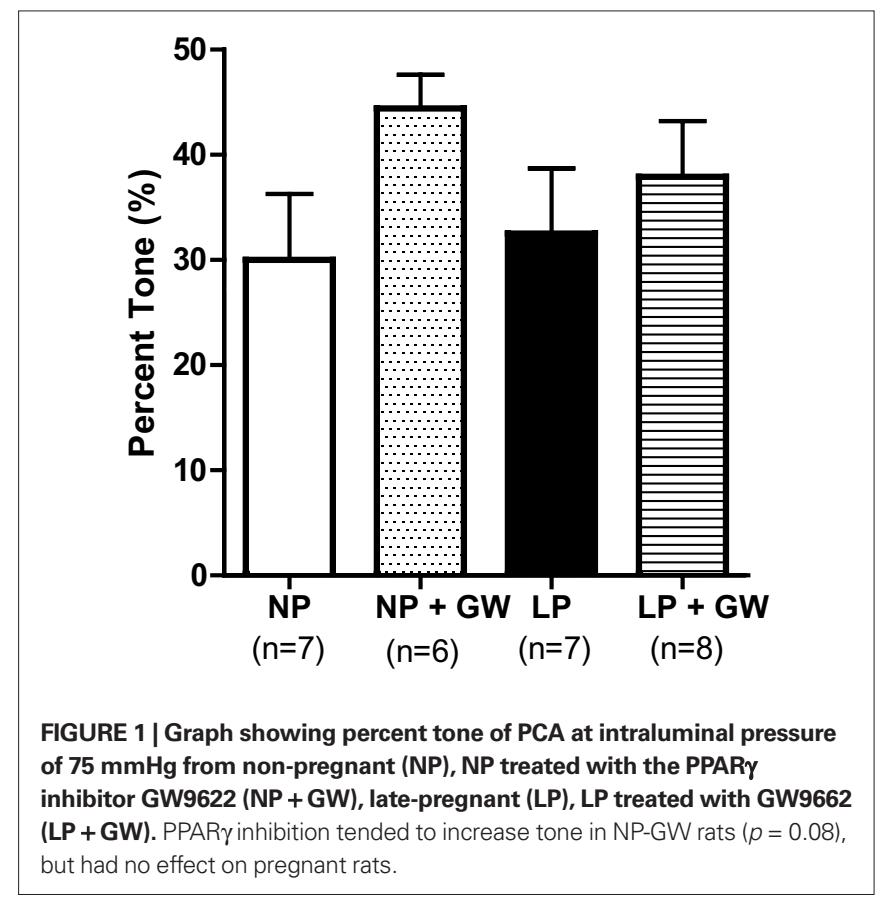


A

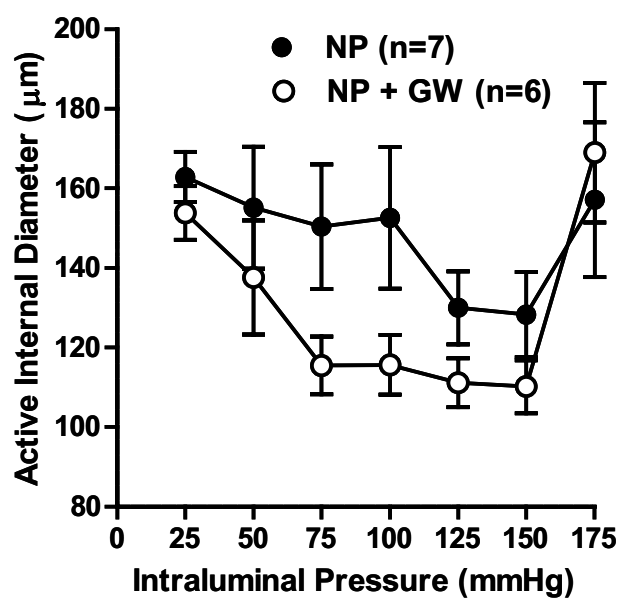

FIGURE 2 | Graph showing active internal diameters versus intraluminal pressure of PCA in (A) non-pregnant (NP) and GW9662-treated NP (NP + GW) groups; (B) late-pregnant (LP) and GW9662-treated LP (LP + GW)
B

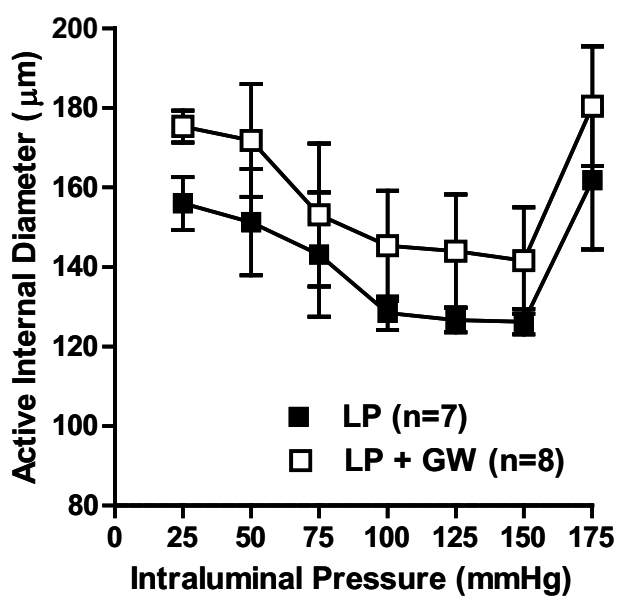

groups. Myogenic activity in PCA was demonstrated by constriction in response to increases in pressure and tended to be increased in NP-GW but not in PCA from LP or LP-GW rats.

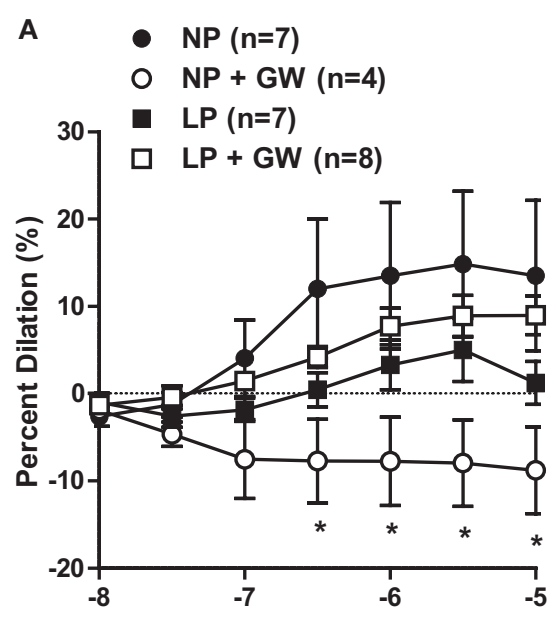

FIGURE 3 | Graph showing percent dilation of PCA in response to (A) ACh $\left(10^{-8}-10^{-5} \mathrm{M}\right)$ and $(B)$ SNP $\left(10^{-8}-10^{-5} \mathrm{M}\right)$ from non-pregnant (NP), NP treated with the PPAR $\gamma$ inhibitor GW9622 (NP + GW), late-pregnant (LP), LP treated

\section{B}

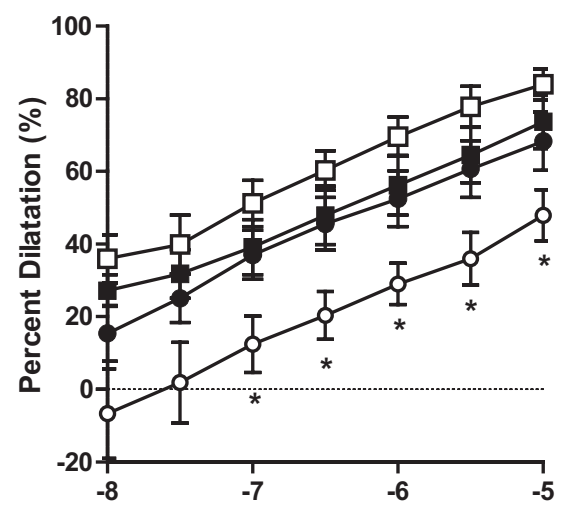

with GW9662 (LP+ GW). Note that two-way ANOVA revealed that dilation to ACh $\left(3 \times 10^{-7}-10^{-5} \mathrm{M}\right)$ and SNP $\left(10^{-7}-10^{-5} \mathrm{M}\right)$ in PCA from NP + GW rats was significantly different from that of $\mathrm{LP}+\mathrm{GW}$ rats. ${ }^{*} p<0.05 \mathrm{vs}$. $\mathrm{LP}+\mathrm{GW}$. revealed that dilator responses to $\mathrm{SNP}$ (from $10^{-7}$ to $10^{-5} \mathrm{M}$ ) in PCA from NP-GW rats were significantly different from that of LP-GW rats. Dilation to SNP in PCA from NP-GW rats was the main contributor to the difference, because GW9662 did not affect dilation to SNP in PCA from LP rats.

\section{EFFECTS OF GW9662 DURING PREGNANCY ON VASCULAR STRUCTURE IN PCA}

To examine structural changes of PCA in response to pregnancy and GW9662 treatment, we measured ID and WT in fully relaxed PCA (by papaverine and diltiazem) at $5 \mathrm{mmHg}$. Measurements were made at minimal pressure $(5 \mathrm{mmHg}$ ) to avoid vessel collapse and eliminate differences due to changes in distensibility. OD, wall-tolumen ratios, and CSA were also calculated. WT was significantly increased in PCA from GW9662-treated NP rats (Table 2). Compared to untreated NP rats, PCA from NP-GW rats had only a small decrease in ID. Thus, the increased WT was the major contributor to the increased wall-to-lumen ratio. GW9662-induced hypertrophy in PCA from NP rats was confirmed by increased CSA. Moreover, similar increases in WT were observed in PCA from LP-GW rats with increased wall-to-lumen ratio and CSA. Two-way ANOVA revealed that GW9662 treatment significantly altered WT, wall-to-lumen ratio, and CSA compared to untreated NP and LP rats. Compared to untreated LP rats, hypertrophy in PCA from LP-GW rats contributed to an increase in OD because ID was similar. These results suggest that PPAR $\gamma$ inhibition caused inward hypertrophic remodeling in PCA from NP rats, but outward hypertrophic remodeling in PCA from LP rats. 
Table 2 | Passive structural measurements of cerebral posterior arteries in non-pregnant (NP), NP treated with GW9662 (NP + GW), latepregnant (LP), and LP treated with GW9662 (LP + GW) at $5 \mathrm{mmHg}$.

\begin{tabular}{|c|c|c|c|c|}
\hline & $\mathrm{NP}(n=8)$ & $\begin{array}{l}\mathrm{NP}+\mathrm{GW} \\
(n=7)\end{array}$ & $\operatorname{LP}(n=8)$ & $\begin{array}{l}\mathrm{LP}+\mathrm{GW} \\
(n=8)\end{array}$ \\
\hline $\begin{array}{l}\text { Inner diameter } \\
(\mu \mathrm{m})\end{array}$ & $127 \pm 5$ & $117 \pm 2$ & $121 \pm 3$ & $121 \pm 4$ \\
\hline $\begin{array}{l}\text { Outer diameter } \\
(\mu \mathrm{m})\end{array}$ & $168 \pm 5$ & $166 \pm 2$ & $164 \pm 4$ & $170 \pm 5$ \\
\hline $\begin{array}{l}\text { Wall thickness } \\
(\mu \mathrm{m})\end{array}$ & $20.5 \pm 0.8$ & $24.3 \pm 0.8^{*}$ & $21.9 \pm 0.9$ & $24.1 \pm 1.1^{*}$ \\
\hline $\begin{array}{l}\text { Wall-to-lumen } \\
\text { ratio }\end{array}$ & $0.162 \pm 0.008$ & $0.208 \pm 0.009^{*}$ & $0.182 \pm 0.008$ & $0.199 \pm 0.008 *$ \\
\hline $\mathrm{CSA}\left(\mu \mathrm{m}^{2}\right)$ & $9542 \pm 535$ & $10786 \pm 355^{*}$ & $9817 \pm 569$ & $11093 \pm 736^{*}$ \\
\hline
\end{tabular}

Data are presented as means \pm SEM.

CSA, cross-sectional area.

${ }^{*} p<0.05$ vs. NP.
To further examine structural changes of the vessel wall in response to pregnancy and GW9662 treatment, we calculated passive distensibility at various intraluminal pressures. Figure 4 shows passive distensibility from all groups of animals. GW9662 treatment significantly increased distensibility at pressures of $30,40,50 \mathrm{mmHg}$ in PCA from NP rats (Figure 4A) but did not affect distensibility of PCA from LP rats (Figure 4B). In addition, compared to NP rats, distensibility was increased at 30, 40, 50, $75 \mathrm{mmHg}$ in PCA from LP rats (Figure 4C). The effect of GW9662 treatment on distensibility was similar in PCA from NP and LP rats (Figure 4D).

\section{EFFECTS OF GW9662 AND PREGNANCY ON TARGET GENE EXPRESSION}

Relative mRNA expression of PPAR $\gamma$ in cerebral vessels was significantly decreased during pregnancy regardless of GW9662 treatment (Figure 5A). GW9662 did not change the expression of PPAR $\gamma$ in cerebral vessels from NP or LP rats. Messenger RNA expression of AT1R expression was significantly decreased in cerebral vessels from LP rats, but was also unaffected by GW9662 treatment in cerebral vessels from NP or LP rats (Figure 5B).
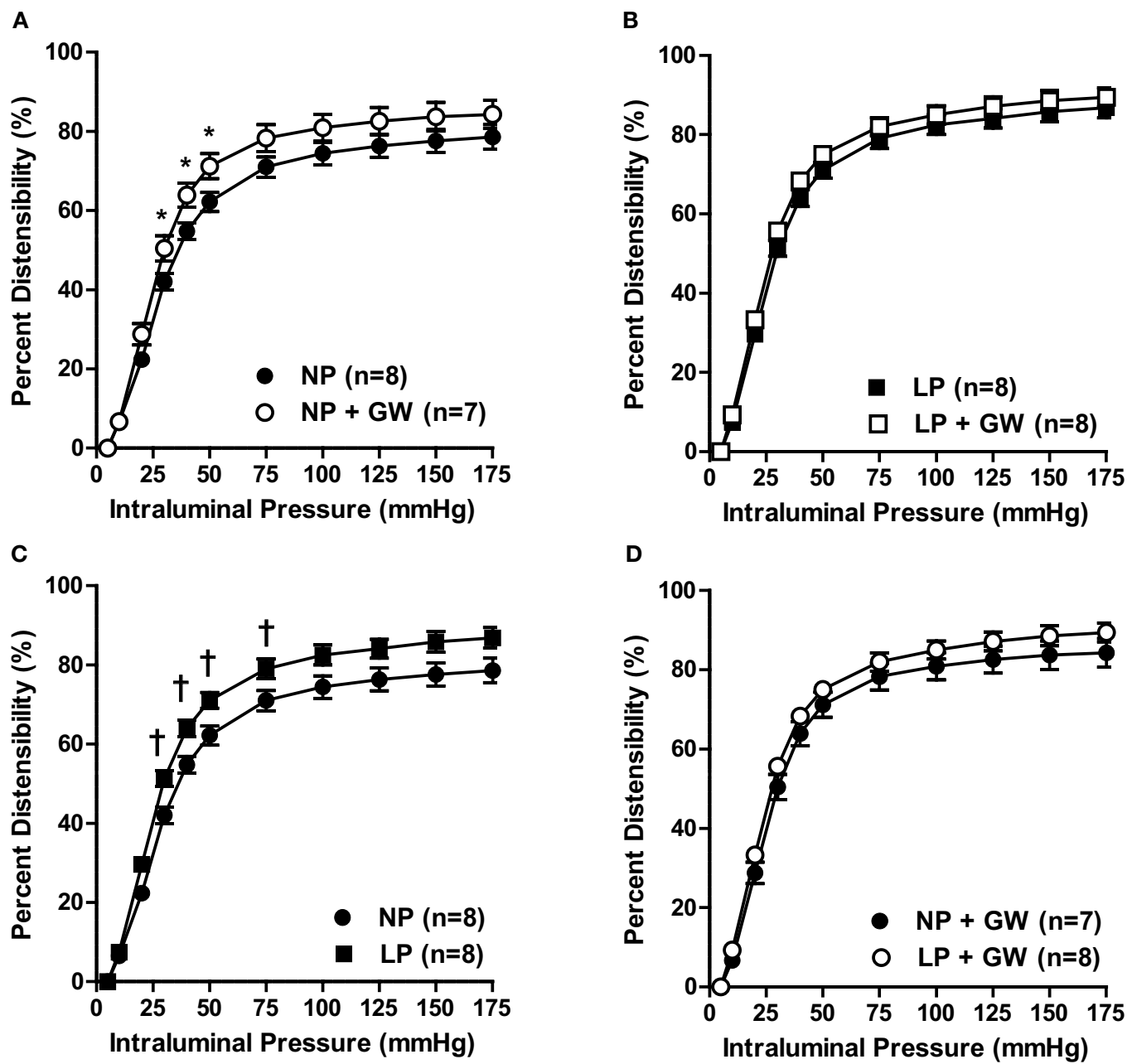

FIGURE 4 | Graph showing passive distensibility versus intraluminal pressure of PCA in (A) non-pregnant (NP) and GW9662-treated NP (NP + GW) groups: (B) late-pregnant (LP) and GW9662-treated LP (LP + GW) groups; (C) NP and LP groups; and (D) NP + GW and LP + GW groups. ${ }^{*} p<0.05$ : NP vs. NP + GW; $+p<0.05:$ NP vs. LP. 


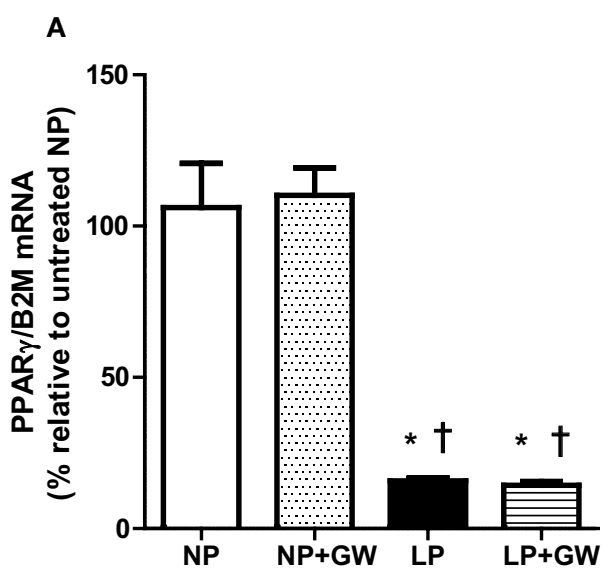

FIGURE 5 | Graph showing relative changes in mRNA expression of (A) PPAR $\gamma$ and (B) angiotensin type 1 receptors (AT1R) in cerebral vessels of non-pregnant (NP), NP + GW9662 (NP + GW), late-pregnant (LP), and LP + GW9662 (LP + GW) groups. Results are presented as percent change in
B

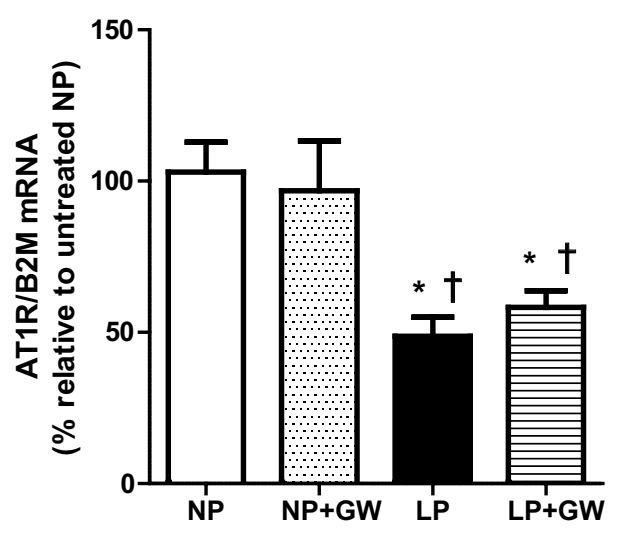

gene expression normalized to $\beta 2$-microglobulin (B2M), an endogenous reference gene, and relative to the untreated NP group ( $n=7-8$, mean \pm SEM). Expression of PPAR $\gamma$ and AT1R was decreased in cerebral vessels from LP rats but unaffected by GW9662 treatment. ${ }^{*} p<0.05$ vs. NP, $+p<0.05$ vs. NP $+\mathrm{GW}$.

\section{DISCUSSION}

There are several novel findings in the present study. First, PPAR $\gamma$ inhibition with GW9662 abolished dilation to ACh and reduced the dilatory response to SNP in PCA from NP rats, suggesting that PPAR $\gamma$ inhibition may cause endothelial dysfunction and reduced smooth muscle responsiveness to NO. However, PPAR $\gamma$ inhibition had no effect on the dilation to ACh and SNP in PCA from LP rats. Second, PPAR $\gamma$ inhibition tended to increase basal tone and myogenic activity in PCA from NP rats. These effects were not seen in PCA from LP rats. Third, PPAR $\gamma$ inhibition increased WT, wall-to-lumen ratio, CSA, and passive distensibility in PCA from NP rats. A small decrease in ID was associated with increased WT, suggesting inward remodeling in these vessels. PPAR $\gamma$ inhibition also increased WT and CSA in PCA from LP rats. However, a small increase in OD was observed in these vessels, suggesting outward remodeling. Lastly, gene expression of PPAR $\gamma$ and AT1R in cerebral vessels were decreased in LP rats regardless of GW9662 treatment. In summary, PPAR $\gamma$ inhibition caused vascular dysfunction and inward remodeling in cerebral arteries from NP animals, but had little effect during pregnancy except to promote modest outward remodeling.

There may be several reasons why GW9662 treatment had little effect on physiological parameters and vascular function in PCA from LP rats. First, pregnancy significantly decreased (by 85\%) gene expression of PPAR $\gamma$, regardless of GW9662 treatment. It is therefore possible that further inhibition of PPAR $\gamma$ that was already highly down-regulated during pregnancy was not efficacious. Second, it is possible that treatment with GW9662 during pregnancy was too mild to have a significant effect. Because we were concerned about PPAR $\gamma$ inhibition interfering with implantation and establishment of pregnancy, we treated animals during the second half of pregnancy. PPAR $\gamma$ inhibition may have had a greater influence if treatment with GW9662 was started in early pregnancy or even prior to pregnancy. In addition, the dose of GW9662 used may not have been high enough to inhibit PPAR $\gamma$ in late-pregnancy when PPAR $\gamma$ is highly activated (Waite et al., 2005). Lastly, PPAR $\gamma$ may still be activated during pregnancy even in the presence of GW9662. Relaxin, a hormone circulating at high concentration during pregnancy, has been shown to activate PPAR $\gamma$ through a non-receptor-mediated mechanism (Singh and Bennett, 2010). Thus, it is possible that other non-receptor-mediated effects of PPAR $\gamma$ may still be present because GW9662 only inhibited receptor-mediated actions of PPAR $\gamma$.

PPAR $\gamma$ has been shown to have a significant beneficial role in protecting the vasculature, especially with regards to endothelial function. Genetic interference with PPAR $\gamma$ function has been shown to decrease ACh-induced dilation in cerebral vessels of male mice (Beyer et al., 2008), suggesting PPAR $\gamma$ activity is important for normal endothelial function in the cerebral circulation. In the present study, PPAR $\gamma$ inhibition with GW9662 treatment decreased ACh and SNP dilation in PCA from NP rats, which is in partial agreement with a previous study (Beyer et al., 2008). Although this previous study did not find an effect of PPAR $\gamma$ on dilation to SNP, another study has shown that interference with PPAR $\gamma$ decreased dilation to SNP in aorta (Halabi et al., 2008). Together, these results suggest that PPAR $\gamma$ inhibition with GW9662 impacts NO-mediated dilation in both endothelium and smooth muscle in males and NP females. However, NO responsiveness was unaffected when PPAR $\gamma$ was inhibited during pregnancy, possibly due to a pregnancy-specific adaptation such as decreased PPAR $\gamma$ expression or a difference in treatment efficacy.

An important function of cerebral arteries is that they constrict or dilate in response to increases or decreases in pressure, respectively. This myogenic activity is well-developed in the brain circulation and underlies autoregulation of cerebral blood flow (Johnson, 1989). In the present study, we tested how PPAR $\gamma$ inhibition during pregnancy affected tone and myogenic activity in PCA. Pregnancy alone did not change basal tone in PCA at physiological pressures $(75 \mathrm{mmHg}$ ), which is similar to our previous study (Cipolla et al., 2004). PPAR $\gamma$ inhibition tended to increase tone in PCA from NP rats, but not in PCA from LP rats. The increased tone in NP-GW rats may be due to reduced endothelium-dependent 
vasodilator production as ACh responses were abolished in those animals, however, we did not directly examine the effect of PPAR $\gamma$ inhibition on basal endothelial vasodilator release. In addition, as dilation to SNP was also decreased in PCA from NP-GW rats, it is possible that the smooth muscle response to vasodilator was also affected by PPAR $\gamma$ inhibition. In addition, PCA from all groups developed myogenic activity as demonstrated by active constriction in response to increases in pressure. Pregnancy did not significantly change myogenic activity within the myogenic pressure range, which agrees with our previous study (Cipolla et al., 2004). Moreover, PPAR $\gamma$ inhibition with GW9662 appeared to increase myogenic activity at lower pressures $(50,75 \mathrm{mmHg})$ in PCA from NP rats, but did not seem to affect myogenic activity from LP rats. Although PPAR $\gamma$ appeared to be involved in regulating tone and myogenic activity in the NP state possibly through endothelium or smooth cell effects, the results from the present study suggest that PPAR $\gamma$ appears to have little effect on basal tone and myogenic activity in cerebral vasculature during pregnancy.

One of the most profound influences of PPAR $\gamma$ in the vasculature is on structural remodeling. Some studies have shown that PPAR $\gamma$ is involved in vascular remodeling in cerebral vessels in male rats (Beyer et al., 2008; Halabi et al., 2008). Pregnancy also has a significant effect in cerebral artery remodeling, especially during hypertension, that is similar to those attributed to PPAR $\gamma$. For example, hypertension can cause inward remodeling in the cerebral vasculature (Baumbach et al., 1988) and pregnancy has been shown to both prevent and reverse hypertensive remodeling in PCA (Cipolla et al., 2006, 2008). In a recent study, similar prevention of hypertensive remodeling of PCA from NP rats was shown in response to PPAR $\gamma$ activation (Cipolla et al., 2010). In the present study, we determined if PPAR $\gamma$ activity during pregnancy impacted structural remodeling in cerebral arteries. PPAR $\gamma$ inhibition caused hypertrophy in PCA from both NP and LP rats, as evidenced by increased WT and CSA. These results suggest that PPAR $\gamma$ may have a role in suppressing smooth muscle growth and migration (Meredith et al., 2009). Interestingly, the remodeling in PCA from NP rats appeared to be different from that of PCA from LP rats. For example, PPAR $\gamma$ inhibition caused inward hypertrophic remodeling in PCA from NP rats but outward hypertrophic remodeling in PCA from LP rats. The differential response to PPAR $\gamma$ inhibition in NP and LP rats is not clear, but may involve pregnancy-induced decrease in AT1R in the cerebral vasculature. Angiotensin II has a significant role in remodeling in cerebral vasculature (Baumbach et al., 2003; Dupuis et al., 2005) through NADPH oxidase-derived superoxide and not hypertension per se (Chan and Baumbach, 2009). This effect of angiotensin II is largely through the activation of AT1R (Griendling et al., 1997). A previous study showed that inward remodeling of cerebral arterioles induced by interference with PPAR $\gamma$ function involved increased superoxide (Beyer et al., 2008). Moreover, PPAR $\gamma$ activation suppresses AT1R in vascular smooth muscle cells (Takeda et al., 2000) and inhibits the signaling involved

\section{REFERENCES}

Amburgey, O. A., Reeves, S. A., Bernstein, I. M., and Cipolla, M. J. (2009). Resistance artery adaptation to pregnancy counteracts the

in angiotensin II-mediated vascular remodeling (Benkirane et al., 2006). Thus, it is possible that inhibition of PPAR $\gamma$ in NP rats, without increased blood pressure, may lead to increased oxidative stress and the loss of suppression to AT1R-mediated inward remodeling. However, this inward remodeling was not seen in PCA from LP rats, possibly due to pregnancy-induced down-regulation of AT1R expression.

Changes in vascular WT and extracellular matrix components alter vascular wall distensibility in cerebral vessels (Baumbach et al., 1988). Thus, we examined how vascular remodeling induced by PPAR $\gamma$ inhibition during pregnancy affected passive distensibility of PCA. PPAR $\gamma$ inhibition increased passive distensibility in PCA from NP rats that may be due to smooth muscle growth (hypertrophy) induced by PPAR $\gamma$ inhibition. In smaller resistance vessels such as the PCA, smooth muscle is relatively more abundant and contributes more significantly to the compliance of the wall than that of larger vessels, in which collagen is the major determinant of vascular stiffness (Baumbach et al., 1988). In contrast, passive distensibility was increased in PCA from untreated LP rats, which was not further affected by PPAR $\gamma$ inhibition. It is worth noting that, compared to NP rats, PCA from untreated LP rats increased distensibility without increases in WT and CSA, suggesting that the increased distensibility was not due to smooth muscle growth that was observed in the GW9662-treated NP rats. The increased distensibility in PCA from LP rats may be due to changes in extracellular matrix components of the vessel wall, such as collagen and elastin, which has been shown in mesenteric arteries during pregnancy (Mackey et al., 1992). These results suggest that both PPAR $\gamma$ inhibition and pregnancy increase passive distensibility, but may involve changes in different vascular wall components.

In conclusion, PPAR $\gamma$ inhibition appears to have little impact on the function and structure of cerebral vasculature during pregnancy. This lack of an effect of PPAR $\gamma$ inhibition in cerebral vasculature may be due, at least in part, to down-regulation of PPAR $\gamma$ and AT1R in cerebral arteries during pregnancy. Changes in expression of PPAR $\gamma$ and AT1R suggest that effects of PPAR $\gamma$ and AT1R may be suppressed in the cerebral vasculature, despite their importance in vascular function and structure in other organs during pregnancy. The resulting effect may make the cerebral circulation less responsive to circulating factors and promote constant hemodynamics in the brain during pregnancy.

\section{ACKNOWLEDGMENTS}

We thank Mr. Timothy Hunter, Ms. Meghan Kohlmeyer and the DNA facility at UVM for their technical expertise and help with PCR. We gratefully acknowledge the continued support from the NIH (NS045940 to MJC; HL088245 to NG), American Recovery and Reinvestment Act supplement (NS045940-S1 to MJC), the American Heart Association Established Investigator Award (0540081N to MJC), the Preeclampsia Foundation Vision Award and the Totman Medical Research Trust.

of cerebral arterioles in hypertensive rats. Circ. Res. 62, 56-64.

Baumbach, G. L., Sigmund, C. D., and Faraci, F. M. (2003). Cerebral arteriolar structure in mice overexpressing human renin and angiotensinogen. Hypertension 41, 50-55.

Benkirane, K., Amiri, F., Diep, Q. N., El Mabrouk, M., and Schiffrin, E. L. (2006). PPAR-gamma inhibits ang 
II-induced cell growth via SHIP2 and 4E-BP1. Am. J. Physiol. Heart Circ. Physiol. 290, H390-H397.

Berger, J. P., Akiyama, T. E., and Meinke, P. T. (2005). PPARs: therapeutic targets for metabolic disease. Trends Pharmacol. Sci. 26, 244-251.

Beyer, A. M., Baumbach, G. L., Halabi, C. M., Modrick, M. L., Lynch, C. M., Gerhold, T. D., Ghoneim, S. M., de Lange, W. J., Keen, H. L., Tsai, Y. S., Maeda, N., Sigmund, C. D., and Faraci, F. M. (2008). Interference with PPARgamma signaling causes cerebral vascular dysfunction, hypertrophy, and remodeling. Hypertension $51,867-871$.

Carlin, A., and Alfirevic, Z. (2008). Physiological changes of pregnancy and monitoring. Best Pract. Res. Clin. Obstet. Gynaecol. 22, 801-823.

Chan, S. L., and Baumbach, G. (2009). Nox2 deficiency prevents angiotensin II-induced hypertrophy and inward remodeling in cerebral arterioles. FASEB J. 23, 613.611.

Cipolla, M. J., Bishop, N., Vinke, R. S., and Godfrey, J. A. (2010). PPAR \{gamma\} activation prevents hypertensive remodeling of cerebral arteries and improves vascular function in female rats. Stroke 41, 1266-1270.

Cipolla, M. J., and Bullinger, L. (2008). Pregnancy decreases cerebrovascular resistance and increases blood-brain barrier permeability during acute hypertension: a role in eclampsia? Reprod. Sci. 15, 288A.

Cipolla, M. J., DeLance, N., and Vitullo, L. (2006). Pregnancy prevents hypertensive remodeling of cerebral arteries: a potential role in the development of eclampsia. Hypertension 47, 619-626.

Cipolla, M. J., Smith, J., Bishop, N., Bullinger, L. V., and Godfrey, J. A. (2008). Pregnancy reverses hypertensive remodeling of cerebral arteries. Hypertension 51, 1052-1057.

Cipolla, M. J., Smith, J., Kohlmeyer, M. M., and Godfrey, J. A. (2009). SKca and IKca channels, myogenic tone, and vasodilator responses in middle cerebral arteries and parenchymal arterioles: effect of ischemia and reperfusion. Stroke 40, 1451-1457.

Cipolla, M. J., Vitullo, L., and McKinnon, J. (2004). Cerebral artery reactivity changes during pregnancy and the postpartum period: a role in eclampsia? Am. J. Physiol. Heart Circ. Physiol. 286, H2127-H2132.

Clapp, J. F. 3rd, and Capeless, E. (1997). Cardiovascular function before, during, and after the first and subsequent pregnancies. Am. J. Cardiol. 80, 1469-1473.

Cox, B. E., Rosenfeld, C. R., Kalinyak, J.E., Magness, R. R., and Shaul, P.W. (1996). Tissue specific expression of vascular smooth muscle angiotensin II receptor subtypes during ovine pregnancy. Am. J. Physiol. 271, H212-H221.

Dupuis, F., Atkinson, J., Liminana, P., and Chillon, J. M. (2005). Comparative effects of the angiotensin II receptor blocker, telmisartan, and the angiotensin-converting enzyme inhibitor, ramipril, on cerebrovascular structure in spontaneously hypertensive rats. J. Hypertens. 23, 1061-1066.

Gordon, M. (2007)."Maternal physiology," in Obstetrics - Normal and Problem Pregnancies, eds S. N. Gabbe Jr and J. L. Simpson (New York: Churchill Liverstone), 55-84.

Griendling, K. K., Ushio-Fukai, M., Lassegue, B., and Alexander, R. W. (1997). Angiotensin II signaling in vascular smooth muscle. New concepts. Hypertension 29, 366-373.

Halabi, C. M., Beyer, A. M., de Lange, W. J., Keen, H. L., Baumbach, G. L., Faraci, F. M., and Sigmund, C. D. (2008). Interference with PPARgamma function in smooth muscle causes vascular dysfunction and hypertension. Cell Metab. 7, 215-226.

Hsueh, W. A., and Law, R. E. (2001). PPARgamma and atherosclerosis: effects on cell growth and movement. Arterioscler. Thromb. Vasc. Biol. 21, 1891-1895.

Johnson, P. C. (1989). The myogenic response in the microcirculation and its interaction with other control systems. J. Hypertens. Suppl. 7, S33-S39; discussion S40.

Learmont, J. G., Cockell, A. P., Knock, G. A., and Poston, L. (1996). Myogenic and flow-mediated responses in isolated mesenteric small arteries from pregnant and nonpregnant rats. Am. J. Obstet. Gynecol. 174, 1631-1636.

Leesnitzer, L. M., Parks, D. J., Bledsoe, R. K., Cobb, J.E., Collins, J. L., Consler, T. G., Davis, R. G., Hull-Ryde, E. A., Lenhard, J. M., Patel, L., Plunket, K. D., Shenk, J. L., Stimmel, J. B., Therapontos, C., Willson, T. M., and Blanchard, S. G. (2002). Functional consequences of cysteine modification in the ligand binding sites of peroxisome proliferator activated receptors by GW9662. Biochemistry 41, 6640-6650.

Livak, K. J., and Schmittgen, T. D. (2001). Analysis of relative gene expression data using real-time quantitative $\mathrm{PCR}$ and the 2(-delta delta $\mathrm{c}(\mathrm{t}))$ method. Methods 25, 402-408.

Mackey, K., Meyer, M. C., Stirewalt, W. S., Starcher, B. C., and McLaughlin, M. K. (1992). Composition and mechanics of mesenteric resistance arteries from pregnant rats. Am. J. Physiol. 263, R2-R8.

Meredith, D., Panchatcharam, M., Miriyala, S., Tsai, Y. S., Morris, A. J., Maeda, N., Stouffer, G.A., and Smyth, S. S. (2009). Dominant-negative loss of PPARgamma function enhances smooth muscle cell proliferation, migration, and vascular remodeling. Arterioscler. Thromb. Vasc. Biol. 29, 465-471.

Palmer, S. K., Zamudio, S., Coffin, C., Parker, S., Stamm, E., and Moore, L. G. (1992). Quantitative estimation of human uterine artery blood flow and pelvic blood flow redistribution in pregnancy. Obstet. Gynecol. 80 1000-1006.

Parent, A., Schiffrin, E. L., and St-Louis, J. (1990). Role of the endothelium in adrenergic responses of mesenteric artery rings of pregnant rats. Am. J. Obstet. Gynecol. 163, 229-234.

Pascoal, I. F., Lindheimer, M. D., Nalbantian-Brandt, C., and Umans, J. G. (1995). Contraction and endothelium-dependent relaxation in mesenteric microvessels from pregnant rats. Am. J. Physiol. 269, H1899-H1904.

Ralevic, V., and Burnstock, G. (1996). Mesenteric arterial function in the rat in pregnancy: role of sympathetic and sensory-motor perivascular nerves, endothelium, smooth muscle, nitric oxide and prostaglandins. Br. J. Pharmacol. 117, 1463-1470.

Robinson, E., and Grieve, D. J. (2009). Significance of peroxisome proliferator-activated receptors in the cardiovascular system in health and disease. Pharmacol. Ther. 122, 246-263.

Sigmund, C. D. (2010). Endothelial and vascular muscle PPARgamma in arterial pressure regulation: lessons from genetic interference and deficiency. Hypertension 55, 437-444.

Singh, S., and Bennett, R. G. (2010) Relaxin signaling activates peroxisome proliferator-activated receptor gamma. Mol. Cell Endocrinol. 315, 239-245.

Sivarajah, A., McDonald, M. C., and Thiemermann, C. (2005). The cardioprotective effects of preconditioning with endotoxin, but not ischemia, are abolished by a peroxisome proliferator-activated receptorgamma antagonist. J. Pharmacol. Exp. Ther. 313, 896-901.

Takeda, K., Ichiki, T., Tokunou, T., Funakoshi, Y., Iino, N., Hirano, K., Kanaide, H., and Takeshita, A. (2000). Peroxisome proliferator-activated receptor gamma activators downregulate angiotensin II type 1 receptor in vascular smooth muscle cells. Circulation 102, 1834-1839.

Waite, L. L., Louie, R. E., and Taylor, R. N. (2005). Circulating activators of peroxisome proliferator-activated receptors are reduced in preeclamptic pregnancy. J. Clin. Endocrinol. Metab. 90, 620-626.

Waite, L. L., Person, E. C., Zhou, Y., Lim, K. H., Scanlan, T. S., and Taylor, R. N. (2000). Placental peroxisome proliferator-activated receptorgamma is up-regulated by pregnancy serum. J. Clin. Endocrinol. Metab. 85, 3808-3814.

Wieser, F., Waite, L., Depoix, C., and Taylor, R. N. (2008). PPAR action in human placental development and pregnancy and its complications. PPAR Res. 2008, 527048.

Williams, K. P., and Wilson, S. (1998). Variation in cerebral perfusion pressure with different hypertensive states in pregnancy. Am. J. Obstet. Gynecol. 179, 1200-1203.

Conflict of Interest Statement: The authors declare that the research was conducted in the absence of any commercial or financial relationships that could be construed as a potential conflict of interest.

Received:28 May 2010; paperpending published: 16 July 2010; accepted: 09 August 2010; published online: 24 August 2010. Citation: Chan S-L, Chapman AC, Sweet JG, Gokina NI and Cipolla MJ (2010) Effect of PPAR $\gamma$ inhibition during pregnancy on posterior cerebral artery function and structure. Front. Physio. 1:130. doi: 10.3389/ fphys.2010.00130

This article was submitted to Frontiers in Vascular Physiology, a specialty of Frontiers in Physiology.

Copyright $\odot 2010$ Chan, Chapman, Sweet Gokina and Cipolla. This is an open-access article subject to an exclusive license agreement between the authors and the Frontiers Research Foundation, which permits unrestricted use, distribution, and reproduction in any medium, provided the original authors and source are credited. 